Article

\title{
In Vitro Analysis of Pyrogenicity and Cytotoxicity Profiles of Flex Sensors to be Used to Sense Human Joint Postures
}

\author{
Giovanni Saggio $^{1, *}$, Luigi Bianchi ${ }^{2}$, Silvia Castelli ${ }^{3}$, Marilina B. Santucci ${ }^{3}$, Maurizio Fraziano ${ }^{3}$ \\ and Alessandro Desideri ${ }^{3}$
}

1 Department of Electronic Engineering, University of Tor Vergata, via del Politecnico, 1-00133 Rome, Italy

2 Department of Civil Engineering and Computer Science, University of Tor Vergata, via del Politecnico, 1-00133 Rome, Italy; E-Mail: luigi.bianchi@uniroma2.it

3 Department of Biology, University of Tor Vergata, Via della Ricerca Scientifica, 1-00173 Rome, Italy; E-Mails: cstslv00@uniroma2.it (S.C.); santucci@scienze.uniroma2.it (M.B.S.); Fraziano@bio.uniroma2.it (M.F.); desideri@uniroma2.it (A.D.)

* Author to whom correspondence should be addressed; E-Mail: saggio@uniroma2.it; Tel.: +39-06-7259-7260; Fax: +39-06-2331-4067.

Received: 7 March 2014; in revised form: 30 May 2014 / Accepted: 25 June 2014 / Published: 1 July 2014

\begin{abstract}
Flex sensors can be usefully adopted as mechanical-electrical transducers to measure human joint movements, since their electrical resistance varies proportionally to the angle assumed by the joint under measure. Over time, these sensors have been investigated in terms of mechanical and electrical behavior, but no reports have detailed the possibility of their adoption not just on top but under the human skin of the joint. To this aim, our work investigated in vitro the pyrogenic potential and cytotoxicity of some commercially available flex sensors as a first step toward the necessary requirements regarding their biocompatibility, to predict possible foreign body reactions when used in vivo. Results demonstrated that some specific flex sensors satisfy such requirements.
\end{abstract}

Keywords: flex sensors; pyrogenic potential; cytotoxicity; biocompatibility; human joints 


\section{Introduction}

Flex sensors are widely used to sense the static and dynamic postures of human joints, transducing angles into electrical resistance values. The reason for their usage relies on their good reliability and the repeatability of the measurements [1], in spite of their lightness and cost effectiveness. Their main application has been the sensing of flex/extension and abdu/adduction movements of the hand's fingers [2]. However, they have been also used to measure rotations of other human joints, such as the elbow [3] and ankle [4], to reveal the kinematics of the trunk [5] and shoulder [6], or to furnish an aid in gait analysis [7] and orthotic appliances [8]. This is because these sensors can be used to sense bending angles both with fixed and/or changing curvature radius too [9].

Flex sensors have been investigated in terms of their mechanical properties [10], of their electrical quasi-static [11] or fast [12] readouts, and of their electrical response related to their shape [13]. However, as far as we know, there have been no efforts to evaluate their pyrogenic and cytotoxic potential, which we aim to investigate here, to reveal potentially adverse effects, if any.

In this context, a classical adverse effect that occurs, following implantation of biomaterials within the body, is a sterile acute inflammatory response called foreign body reaction. Inflammasome activation and IL- $1 \beta$ release represent the early molecular events leading to the initial recruitment of neutrophils and subsequently of macrophages, which over a period of weeks to months, mediate the next recruitment of fibroblasts releasing collagen, whose final outcome is the encapsulation and isolation of the foreign body [14-17].

Here, we intend to establish the cytotoxic and pyrogenic response of these kinds of sensors as a first step in considering the possibility to use them directly under the human skin, which can be relevant in special applications or events. We refer, for instance, to the possibility of semi-permanent insertion under the skin of the flex sensors for people who experienced severe injuries, with reduction of some motor activities and/or joints' degrees of freedom (DOFs). The semi-permanent insertion can furnish a particular solution in just some special cases, of course, but can sometimes assure a better quality of life, avoiding the permanent usage of external supports embracing the sensors. Under-skin sensor(s) can provide a continuous stream of data, useful to monitor the reduced motor activities, and/or to furnish information about the effectiveness of orthoses, and/or to drive electro-mechanical prosthesis or external motor actuators.

In such a framework, this work intends to evaluate in vitro the cytotoxicity and the pyrogenic potential of some of the most widely used flex sensors. This is also because, as far as we know, no commercially available sensors, capable to measure joint's flexion, exist specifically designed for epicutaneous use.

\section{Materials and Methods}

In this section, we treat of the nature of the used sensors, their electrical behavior, and the way they were handled to determine their pyrogenic potential and cytotoxicity. 


\subsection{Sensors}

Flex sensors are commonly made of brittle ink carbon resistive elements printed on top or within a thin flexible plastic substrate, shaped as a stripe. Micro gaps result within the ink proportionally with flex angles, so that higher flexion produces higher electrical resistance. Two metal terminals realize conductive connections.

Currently, three main brands of flex sensors are affordable and easily available: Spectra Symbol (Salt Lake City, UT, USA), Abrams-Gentile (New York, NY, USA), and Flexpoint Inc. (South Draper, UT, USA). The latter produces flex sensors with the greatest resistance variation and higher measurement repeatability and accuracy, within few degrees [18], when bent; this was the reason to select them for our study. Dimensions of the sensor's strip are approximately $7.1 \mathrm{~mm}$ wide, $0.1 \mathrm{~mm}$ thin with commercial lengths of $2.54 \mathrm{~cm}, 5.08 \mathrm{~cm}$ and $7.62 \mathrm{~cm}$ (Figure 1). In particular, we investigated the $5.08 \mathrm{~cm}$ version in three variants: non-encapsulated, polyester-encapsulated and polymide-encapsulated.

Figure 1. $2.54 \mathrm{~cm}(1 "), 5.08 \mathrm{~cm}(2 ”)$, and $7.62 \mathrm{~cm}$ (3”) long flex sensors by Flexpoint Inc. The darker regions are covered with the carbon based resistive elements. At the left ends the metallic terminals for electric connections.

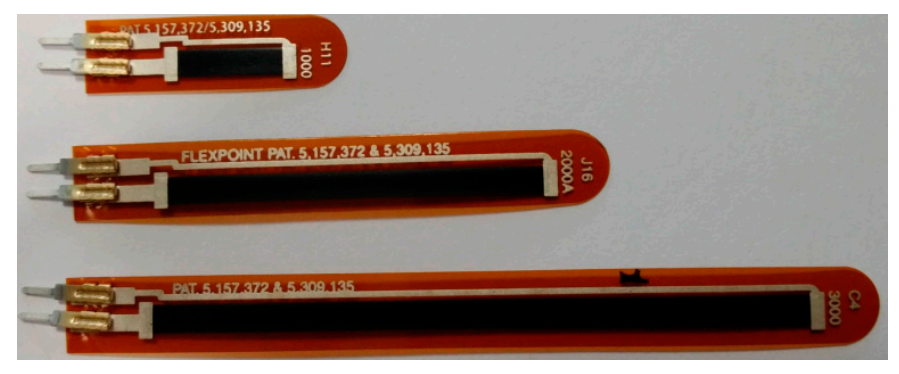

Figure 2. The setup was to measure the electrical behavior of the sensor when flexed to different angles. A stepper motor moves the rotating leaf of a hinge and a multimeter records the resistance value of the sensor. A Labview ${ }^{\circledR}$ routine controls the overall system.

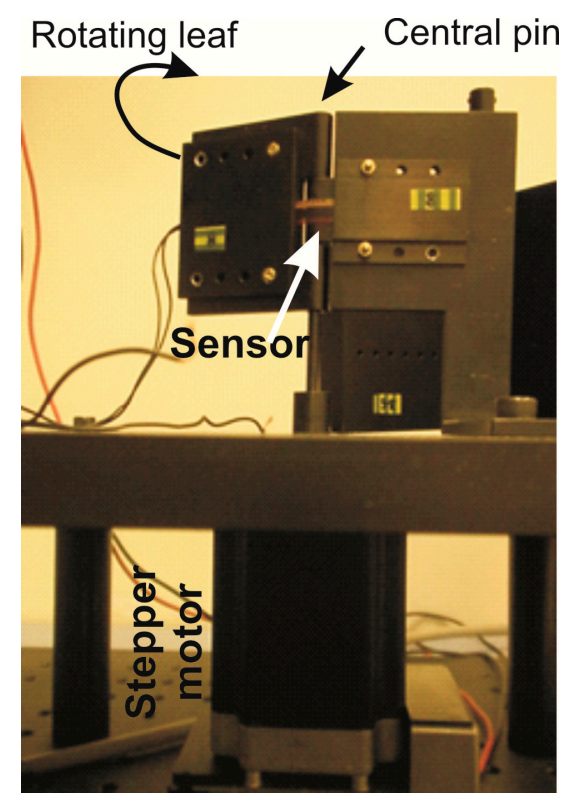


In order to determine their electrical behavior, so as to associate an electrical resistance value to any bending angle, we performed quasi-static measurements using a homemade semi-automatic measurement set-up (Figure 2). It consisted of a hinge with a central pin around which the sensor was automatically flexed by a stepper motor (PD-109-57 by Trinamic, Hamburg, Germany), controlled by a Labview ${ }^{\circledR}$ routine (details reported in [11]). One leaf of the hinge was rotated forwards and backwards from 0 to 90 degrees, stepped 5 degrees, and at each step the new resistance value of the sensor was acquired by a digital multimeter (34405A by Agilent, Santa Clara, CA, USA). No further investigation was necessary, since any other aspects, in particular the accuracy, the reliability [19], and the stability over time [18], were already investigated. Ten measurement cycles were performed on sensors both before and after the pyrogenic and biocompatibility tests, so to compare the respective results in order to evaluate possible changes in their resistance variation $v s$. bending angle, if any.

\subsection{Sensor's Handling for Biological Assays}

We preliminarily washed the sensors with $70 \%$ ethanol and water, then dried and sterilized them under UV light for $30 \mathrm{~min}$, and finally used them for the cell viability and pyrogenic tests. The carbon/plastic part and, in some specific cases the metal part too (details in the following sections), were incubated in Dulbecco's Modified Eagle Medium (DMEM) for three days at $37{ }^{\circ} \mathrm{C}$, to permit the release of the soluble components in the medium, and study their potential toxicity. Foetal bovine serum and L-glutamine can degrade if incubated at $37{ }^{\circ} \mathrm{C}$ over an extended period, for this reason we preferred to add these two components to the culture medium immediately before the experiment.

\subsection{Cell Cultures}

Human keratinocytes (HACAT) were grown in DMEM medium supplemented with 10\% heat-inactivated fetal bovine serum, penicillin (100 units $/ \mathrm{mL})$, streptomycin (100 units $/ \mathrm{mL})$, and $4 \mathrm{mmol} / \mathrm{L}$ glutamine in a humidified atmosphere of $95 \%$ air and $5 \% \mathrm{CO}_{2}$ at $37{ }^{\circ} \mathrm{C}$.

\subsection{Cell Viability Assay}

Cell proliferation was evaluated by using the CellTiter 96 Aqueous One Solution Cell Proliferation Assay (Promega, Madison, WI, USA). The assay used the tetrazolium compound 3-(4,5-dimethylthiazol-2-yl)-5-(3carboxymethoxyphenyl)2-(4-sulphenyl)-2H-tetrazolium, inner salt (MTT). In particular, 104 cells were seeded in 96 well plates in the presence of a medium previously incubated with the only plastic part of polyester, polyimide and no-encapsulated sensors, and grown for $24 \mathrm{~h}$. Later, the cell proliferation was monitored adding $20 \mu \mathrm{L} /$ well of MTT for $2 \mathrm{~h}$ at $37^{\circ} \mathrm{C}$ in a humidified, 5\% $\mathrm{CO}_{2}$ atmosphere. The absorbance at $490 \mathrm{~nm}$ was recorded using an ELISA plate reader [20]. The final value represented the mean $\pm \mathrm{SD}$ of three replicates. As a positive control of the assay, we used cells grown in fresh DMEM medium.

\subsection{Pyrogenic Potential in Whole Blood Assay}

The release of IL-1 $\beta$ was used to test the biocompatibility of the sensors since their release is known to be directly associated with the biocompatibility of medical devices [21]. 
Briefly, blood was drawn from three healthy volunteers ( 2 males, 1 female) into heparinised tubes. Polyester, polyimide and non-encapsulated sensors were incubated in 24 wells plates, with $100 \mu \mathrm{L}$ of fresh blood diluted in PBS, in a total volume of $1 \mathrm{~mL}$ for $24 \mathrm{~h}$ at $37{ }^{\circ} \mathrm{C}$ and $5 \% \mathrm{CO}_{2}$. As positive control, blood was stimulated with $100 \mathrm{ng} / \mathrm{mL}$ Lipopolysaccaride (LPS), whereas as negative control the blood was not stimulated. Cell-free supernatants were collected and stored at $-80{ }^{\circ} \mathrm{C}$ until use. The release of IL-1 $\beta$ in the supenatant was detected by ELISA by a commercially available kit (Thermo Scientific), and used according to the manufacturer's instructions.

\section{Results}

\subsection{Cytotoxicity}

Cell proliferation assays were carried out to estimate the cytotoxicity of the different regions/materials of the sensor. To this aim, the different sensors were incubated in DMEM for three days, as above described, and we used the MTT (3-(4,5-dimethylthiazol-2-yl)-5-(3-carboxymethoxyphenyl)2-(4-sulfophenyl)-2H-tetrazolium) assay for cytotoxicity. This is based on the cleavage of the yellow tetrazolium salt MTT to purple formazan crystals in metabolically active cells, the colorimetric signal being proportional to the number of viable cells. The results, shown in Figure 3, indicate that the encapsulated or non-encapsulated sensors do not show any sign of cytotoxicity, and can be subsequently considered to evaluate their possible pyrogenic effect. On the other hand, the sensors demonstrated high cytotoxicity when their metal parts were incubated with the medium used in the assay, just because of the interaction between the cells and the metallic parts only.

Figure 3. Cells viability evaluated with MTT assay. "Nude", "PE" and "PM" stand respectively for "non-encapsulated", "polyester-encapsulated" and "polymide-encapsulated" sensors. HACAT cells were incubated overnight in medium pre-incubated with: the metal parts, no-encapsulated ("nude") sensor, polyester ("PE") and polyimide ("PM") encapsulated sensors. CTRL-; negative control, cells incubated with 1\% triton-X100. CTRL+; positive control; cells grown in fresh DMEM. Results are indicated as means \pm SD of three different measures.

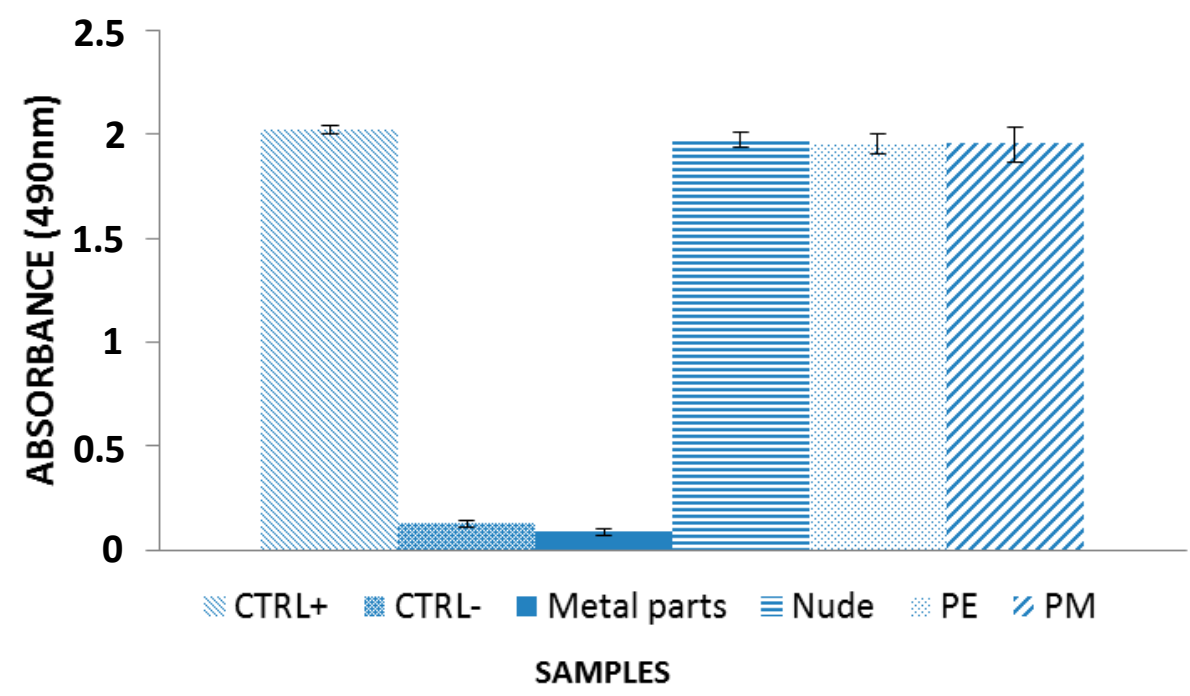




\subsection{Analysis of Pyrogenic Potential of the Different Flex Sensors}

The pyrogenic potential of any biomaterial is an important component of biocompatibility analysis, with mounting evidence showing that IL-1 $\beta$ production by host innate immunity can induce foreign body reaction and result in implant failure [21]. In this context, foreign body reaction was assessed in vitro by evaluating IL- $1 \beta$ production by whole blood samples exposed in vitro to the different flex sensors (the blood came from three healthy volunteers). Human whole blood was brought into contact with the non-encapsulated, polyester or polyimide encapsulated flex sensors and the release of cytokine IL-1 $\beta$, used as an in vitro assay predictive of foreign body reaction, was measured by ELISA assay. The results shown in Figure 4 demonstrate a significant IL-1 $\beta$ release both in non-encapsulated or polyester encapsulated sensors, although significantly lower than that observed in LPS stimulated positive control. The polyester encapsulated sensor showed a lower proinflammatory profile. However, in all tested blood samples, the polyimide-encapsulated flex sensors display an IL-1 $\beta$ release comparable to that of the negative control and allow us to consider it for further pre-clinical studies.

Figure 4. IL-1 $\beta$ release in a whole blood assay. Whole blood obtained by three different healthy donors was collected and incubated overnight in absence (-ve controls) or in the presence of non-encapsulated ("nude"), polyester-encapsulated ("PE") or polyimide-encapsulated ("PM") sensors. +ve controls consisted of LPS. Results are expressed \pm SD of three different culture determinations.

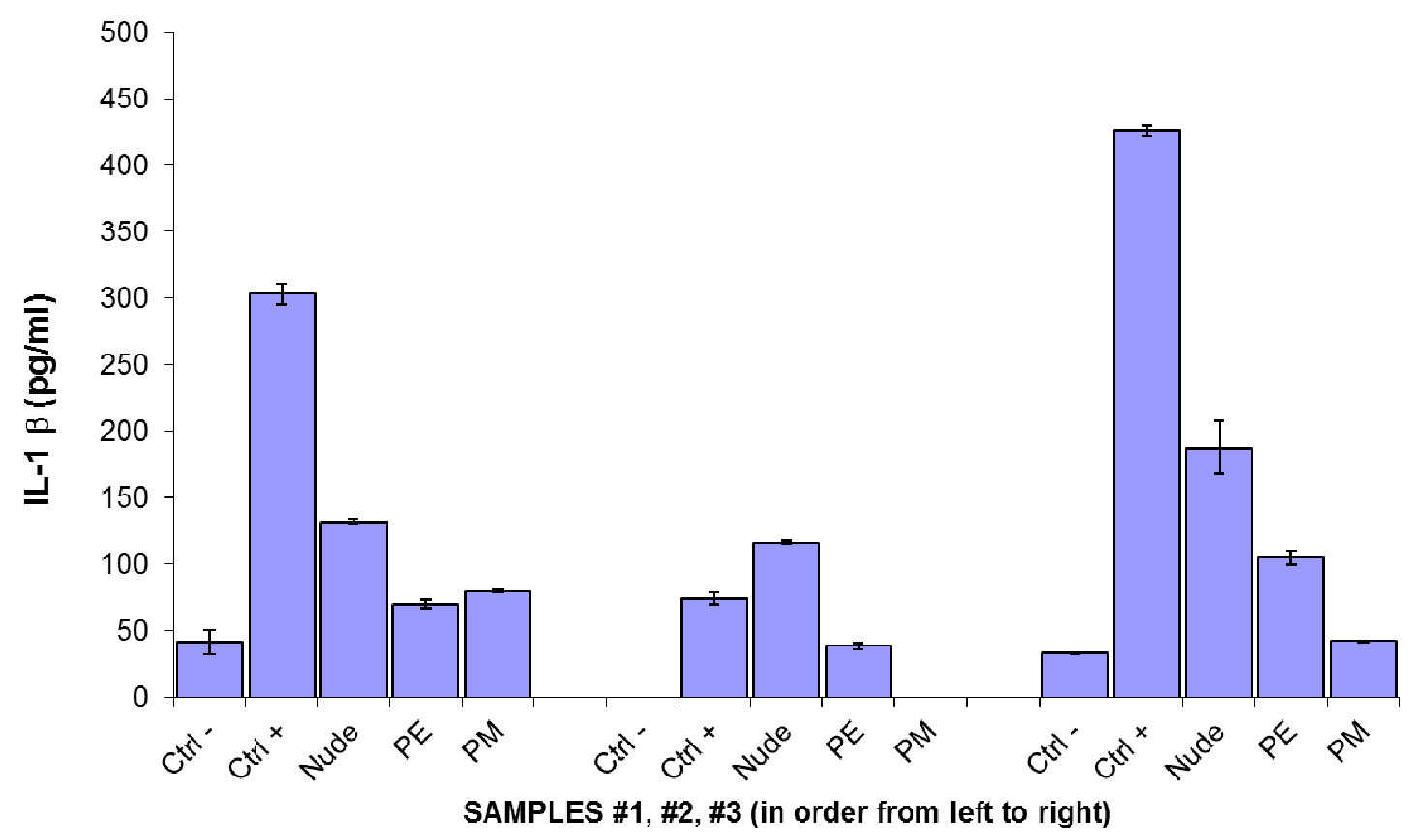

\subsection{Resistance Measurements}

Before and after the in vitro pyrogenicity and cytotoxicity tests, we measured the electrical behavior of the sensors, in terms of resistance $v s$. flex angle pairs, to demonstrate potential degradation of sensor's response following cell culture, if any. Figure 5 shows the electrical behavior of one sample of polymide encapsulated flex sensor, and it is relevant to observe that there is no hysteresis effect and no significant degradation. The same occurred for all the sensors tested. 
Figure 5. Two resistance vs. bending angle characteristics of one sample of polymide-encapsulated 2" flex sensor. The two curves were recorded before and after the tests. There is no evidence of any degradation of the electrical behavior of the sensor. This plot is for just one sample, but the same kind of behavior was recorded for all the sensors evaluated.

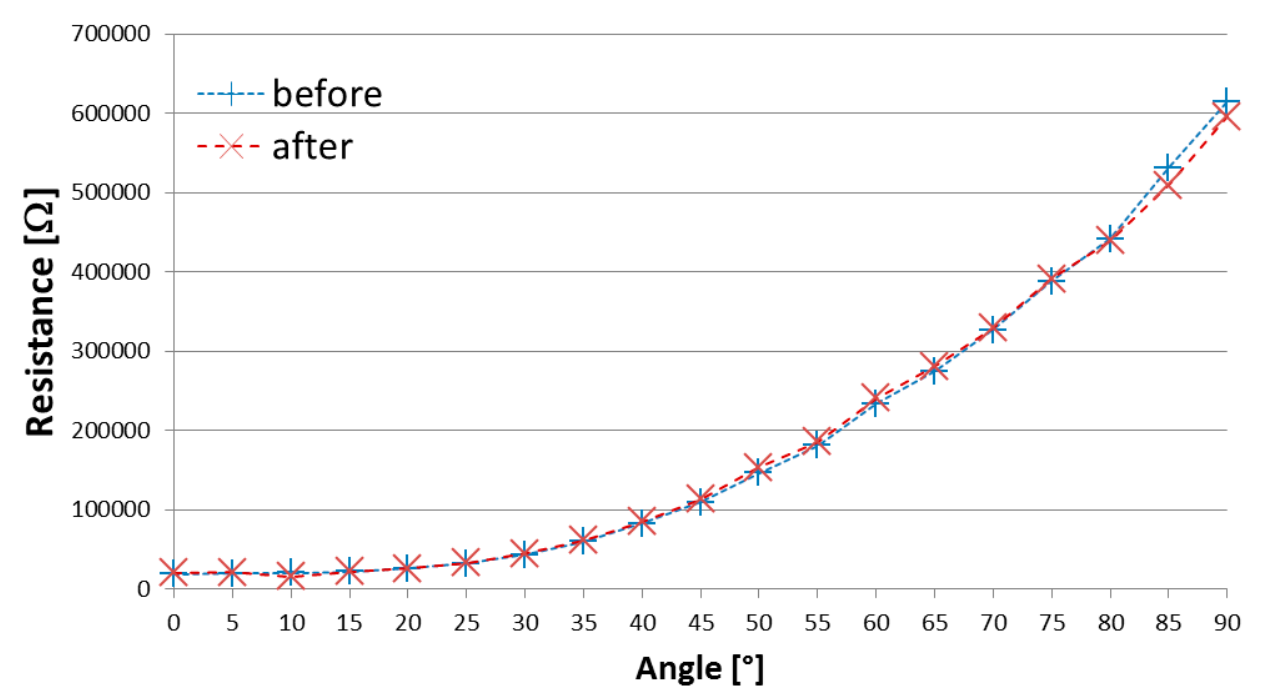

\section{Discussion and Conclusions}

Biomaterials are widely used in regenerative medicine. Worldwide there are over 100 different commercially available biomaterial-based medical devices, with different composition and formulation for clinical use [22]. Although these materials are commonly used and considered relatively biocompatible, a number of studies have shown that they may have biocompatibility issues [23]. In this study, we focused our attention on the pyrogenicity and cytotoxicity profiles of particular devices, such as flex sensors, which are capable of converting mechanical bending effects into an electrical value. In particular, we analyzed the most adopted commercially available ones, never considered before for epicutaneous use. The implantation of such flex sensors into experimental animals is the most effective experiment to define their full biocompatibility, however this work represents a first step to test their capability to induce a direct cytotoxic and/or pyrogenic effect. Permanent cell lines are generally employed for in vitro cytotoxicity, which can be monitored by a MTT assay, according to the international standard ISO 10993-5. Given that the flex sensors described herein should ideally be implanted under the skin, we used a model of keratinocyte cell line in a short-term medium extraction test, where DMEM was incubated with the different sensors for $24 \mathrm{~h}$, and then used to culture HACAT cell line for cytotoxic evaluation after 3 days by MTT assay. Reported results show a complete absence of cytotoxicity in the cell model used. Another parameter to evaluate biocompatibility issues is given by the prediction of foreign body reaction. This reaction is mediated by immune cells, such as leukocytes and platelets, which intervene in order to protect the body from the foreign object [24], and is caused by the tissue injury resulting from implantation of the device, as well as the exposure of danger associated molecular pattern on the surface of the device in the body [25]. The main stages of this process include acute inflammation, chronic inflammation, the formation of granulomatous tissue and the walling off the device by a vascular, collagenous fibrous capsule [26]. This fibrous wall 
confines the implanted device and prevents it from interacting with surrounding tissues thus leading to failure implantation. One of the earlier soluble mediators of foreign body reaction is IL- $1 \beta$, which is immediately released following tissue injury and/or the recognition of danger associated molecular pattern [27] on the surface of the device, and can be used as a predictable marker of inflammatory response [28].

In this study, we used whole blood based assay in order to get information about in vitro reactogenicity of some of the most common flex sensors, by different immune cell types. Because the assay mimics the natural environment, whole blood culture may be the best environment to study cell activation and cytokine production in vitro. In our work, the whole blood based assay was able to discriminate the levels of response induced by three different types of flex sensors, which did not show any sign of cytotoxicity but identified polyimide-encapsulated sensor only as the less reactogenic one, so to become a possible candidate for future experiments of implantation in small animal models. Reasonably, we do not expect a different answer even for longer incubation time (even if further long-term tests will be performed) and so implantation will be the next programmed experiment to verify a full in vivo biocompatibility of the sensor.

In conclusion, the present study reports evidence concerning the in vitro pyrogenic potential and cytotoxicity of some commercially available flex sensors, in order to assess their biocompatibility, and in view of a possible semi-permanent insertion under the skin for people who have experienced severe injuries to continuously monitor their residual motor activities. The study demonstrated that, among the adopted three types of flex sensors, the polyimide-encapsulated one showed the lowest pyrogenic effect, with no relevant cytotoxicity, and displayed a low proinflammatory profile in the discussed experimental models.

Finally, the impact on sensor performances, in terms of changes in the resistance $v s$. bending angle characteristics, was practically negligible, since no electrical mismatches resulted between resistance values measured before and after all the tests. In a spirit of completeness, the authors plan to perform tests of accuracy, reliability and stability over time to determine differences after the treatments, if any. The overall results suggest such flex sensors for consideration in future pre-clinical studies.

\section{Author Contributions}

Giovanni Saggio proposed the original idea, took the leadership on this research work, and realized/utilized the electro-mechanical setup for the electronic measurements. Luigi Bianchi contributed to the electronic measurements and data analyzing. Alessandro Desideri, Silvia Castelli, Marilina B. Santucci and Maurizio Fraziano proposed and realized the framework and the tool development for the pyrogenicity and cytotoxicity tests. All authors contributed to the discussion, to test improvements, to the analysis of results, and to the manuscript writing.

\section{Conflicts of Interest}

The authors declare no conflict of interest. 


\section{References}

1. Simone, L.K.; Kamper, D.G. Design considerations for a wearable monitor to measure finger posture. J. Neuroeng. Rehabil. 2005, 2, 1-10.

2. Dipietro, L.; Sabatini, A.M.; Dario, P. A Survey of Glove-Based Systems and Their Applications. IEEE Trans. Syst. Man Cybern. C 2008, 38, 461-482.

3. Ambar, R.; Bin Mhd Poad, H.; Bin Mohd Ali, A.M.; Ahmad, M.S.; Jamil, M.M.A. Multi-sensor Arm Rehabilitation Monitoring Device. In Proceedings of the Int. Conference on Biomedical Engineering (ICoBE), Penang, Malaysia, 27-28 February 2012; pp. 424-429.

4. Keyfitz, R. Design of a Range of Motion Sensor for Ankle Rehabilitation Monitor. Bachelor Thesis, Department of Electrical and Biomedical Engineering, McMaster University, Hamilton, ON, Canada, 2010.

5. Brush U.A. Design and Validation of an Intensity-Based POF Bend Sensor Applications in Measuring Three-Dimensional Trunk Motion. Ms.c. Thesis, Ohio State University, Columbus, OH, USA, 2010.

6. B Pereira do Carmo, J.P. RF CMOS Transceiver a $2.4 \mathrm{GHz}$ para Microssistemas em Redes de Sensors Sem Fios. Ph.D. Thesis, Universitade do Minho, Braga, Portugal, 2007.

7. Morris Bamberg, S.J.; Benbasat, A.Y.; Scarborough, D.M.; Krebs, D.E.; Paradiso, J.A. Gait Analysis Using a Shoe-Integrated Wireless Sensor System. IEEE Trans. Inf. Technol. Biomed. 2008, 12, 413-423.

8. Yang, W.C.; Huang, W.H.; Chang, C.H.; Chung, K.C. Development of the Portable Posture Training Device. In Proceedings of the ISB XXth Congress-ASB 29th Annual Meeting, Cleveland, OH, USA, 31 July-5 August 2009; p. 240.

9. Orengo, G.; Sbernini, L.; Di Lorenzo, N.; Lagati, A.; Saggio, G. Curvature Characterization of Flex Sensors for Human Posture Recognition. Univers. J. Biomed. Eng. 2013, 1, 10-15.

10. Saggio, G. Mechanical Model of Flex Sensors used to Sense Finger Movements. Sens. Actuators A Phys. 2012, 185, 53-58.

11. Saggio, G. Electrical Resistance Profiling of Bend Sensors Adopted to Measure Spatial Arrangement of the Human Body. In Proceedings of the 4th International Symposium on Applied Sciences in Biomedical and Communication Technologies (ISABEL), Barcelona, Spain, 26-29 October 2011.

12. Orengo, G.; Lagati, A.; Saggio, G. Bend Sensors Modelling for Fast Signal Recovering in Human Motion Analysis. In Proceedings of the 3rd International Conference on Sensor Device Technologies and Applications (SENSORDEVICES), Rome, Italy, 19-24 August 2012; pp. 21-24.

13. Saggio, G.; Lagati, A.; Orengo, G. Shaping Resistive Bend Sensors to Enhance Readout Linearity. ISRN Electron. 2012, 2012, doi:10.5402/2012/359759.

14. Malik, A.F.; Hoque, R.; Ouyang, X.; Ghani, A.; Hong, E.; Khan, K.; Moore, L.B.; Ng, G.; Munro, F.; Flavell, R.A.; et al. Inflammasome components Asc and caspase-1 mediate biomaterial-induced inflammation and foreign body response. Proc. Natl. Acad. Sci. USA 2011, 108, doi:10.1073/pnas.1105152108. 
15. Ekdahl, K.N.; Lambris, J.D.; Elwing, H.; Ricklin, D.; Nilsson, P.H.; Teramura, Y.; Nicholls, I.A.; Nilsson B. Innate immunity activation on biomaterial surfaces: A mechanistic model and coping strategies. Adv. Drug Deliv. Rev. 2011, 63, 1042-1050.

16. Engberg, A.E.; Rosengren-Holmberg, J.P.; Chen, H.; Nilsson, B.; Lambris, J.D.; Nicholls, I.A.; Ekdahl, K.N. Blood protein-polymer adsorption: Implications for understanding complement-mediated hemoincompatibility. J. Biomed. Mater Res. A 2011, 97A, 74-84.

17. Fink, H.; Hong, J.; Drotz, K.; Risberg, B.; Sanchez, J.; Sellborn, A. An in vitro study of blood compatibility of vascular grafts made of bacterial cellulose in comparison with conventionally-used graft materials. J. Biomed. Mater Res. A 2011, 97A, 52-58.

18. Oess, N.P.; Wanek, J.; Curt, A. Design and evaluation of a low-cost instrumented glove for hand function assessment. J. NeuroEng. Rehabil. 2012, 9, 1-11.

19. Gentner, R.; Classen, J. Development and evaluation of a low-cost sensor glove for assessment of human finger movements in neurophysiological settings. J. Neurosci. Methods 2009, 178, $138-147$.

20. Van Meerloo, J.L, Kaspers, G.J.; Cloos, J. Cell sensitivity assays: The MTT assay. Methods Mol. Biol. 2011, 731, 237-245.

21. Cardona, M.A.; Simmons, R.L.; Kaplan, S.S. TNF and IL-1 generation by human monocytes in response to biomaterials. J. Biomed. Mater. Res. 1992, 26, 851-859.

22. Grotenhuis, N.; Bayon, Y.; Lange, J.F.; Van Osch, G.J.V.M.; Bastiaansen-Jenniskens Y.M. A culture model to analyze the acute biomaterial-dependent reaction of human primary macrophages. Biochem. Biophys. Res. Commun. 2013, 433, 115-120.

23. Mendes, S.C.; Reis, R.L.; Bovell, Y.P.; Cunha, A.M.; van Blitterswijk, C.A.; de Bruijn, J.D. Biocompatibility testing of novel starch-based materials with potential application in orthopaedic surgery: A preliminary study. Biomaterials 2001, 22, 2057-2064.

24. Anderson, J.M.; Rodriguez, A.; Chang, D.T. Foreign body reaction to biomaterials. Semin. Immunol. 2008, 20, 86-100.

25. Anderson, J.M. Biological responses to biomaterials. Annu. Rev. Mater. 2001, 31, 81-110.

26. Ratner, B.D.; Bryant, S.J. Biomaterials: Where we have been and where we are going. Annu. Rev. Biomed. Eng. 2004, 6, 41-75.

27. Matzinger, P. Friendly and dangerous signals: Is the tissue in control? Nat. Immunol. 2007, 8, $11-13$.

28. Daneshian, M.; von Aulock, S.; Hartung, T. Assessment of pyrogenic contaminations with validated human whole-blood assay. Nat. Protoc. 2009, 4, 1709-1721.

(C) 2014 by the authors; licensee MDPI, Basel, Switzerland. This article is an open access article distributed under the terms and conditions of the Creative Commons Attribution license (http://creativecommons.org/licenses/by/3.0/). 